\title{
LINEAR STOCHASTIC EQUATIONS IN THE CRITICAL CASE
}

\author{
DARIUSZ BURACZEWSKI AND KONRAD KOLESKO
}

\begin{abstract}
We consider solutions of the stochastic equation $X \stackrel{d}{=} \sum_{i=1}^{N} A_{i} X_{i}+B$, where $N$ is a random natural number, $B$ and $A_{i}$ are random positive numbers and $X_{i}$ are independent copies of $X$, which are independent also of $N, B, A_{i}$. Properties of solutions of this equation are mainly coded in the function $m(s)=\mathbb{E}\left[\sum_{i=1}^{N} A_{i}^{s}\right]$. In this paper we study the critical case when the function $m$ is tangent to the line $y=1$. Then, under a number of further assumptions, we prove existence of solutions and describe their asymptotic behavior.
\end{abstract}

\section{INTRODUCTION}

The main purpose of the present paper is to study a class of linear stochastic equations, existence of their solutions and to describe properties of those solutions. The simplest example we have in mind is the random difference equation, called often also a first order random coefficients autoregressive model,

$$
X \stackrel{d}{=} A X+B
$$

where all the random variables are real valued, $X$ is independent of the pair $(A, B)$, and the sign ' $\underline{\underline{d}}$, denotes equality in distribution. It is well-known that the equation above has a unique solution if $\mathbb{E}[\log A]<0$ and $\mathbb{E}\left[\log ^{+}|B|\right]<\infty$. The celebrated Kesten theorem [19] says that if a random variable $X$ is a solution of the equation (1.1), then under a number of assumptions, the main being existence of a positive $\alpha$ such that $\mathbb{E}\left[A^{\alpha}\right]=1$, the random variable $X$ is $\alpha$-regularly varying, i.e.

$$
\lim _{x \rightarrow \infty} x^{\alpha} \mathbb{P}[X>x]=C_{+},
$$

for some positive constant $C_{+}$(see also the paper of Goldie [15] for a transparent and elegant proof). Since the random difference equation appears both in many applied models e.g. in financial mathematics and in purely mathematical problems, the last result found enormous number of applications in the literature.

In this paper we consider general linear stochastic equations, i.e. equations of the form

$$
X \stackrel{d}{=} \sum_{i=1}^{N} A_{i} X_{i}+B
$$

where $X, X_{i}$ are i.i.d. and independent of $\left(N, B, A_{1}, A_{2} \cdots\right)$. Notice that the last formula depends only on $N$ first values of $A_{i}$ 's, therefore without any loss of generality, we assume that $A_{i}=0$ for $i>N$. Moreover in this paper we restrict our attention to positive random variables, i.e. we assume that $X_{i}, A_{i}$ and $B$ are positive.

2010 Mathematics Subject Classification. Primary 60H25; secondary 60J80, 60F10.

Key words and phrases. Smoothing transform, linear stochastic equation, regular variation, large deviations.

D. Buraczewski was partially supported by MNiSW grant N N201 393937. K. Kolesko was partially supported by MNiSW grant N N201 610740 . 
Equation (1.2) is also called the inhomogeneous smoothing transform and the explanation of this name is the following. Given $\mu \in \mathcal{P}\left(\mathbb{R}^{+}\right)$we define $\widetilde{T} \mu$ as the law of $\sum_{i=1}^{N} A_{i} X_{i}+B$, where $X_{1}, X_{2}, \ldots$ is i.i.d. sequence with distribution $\mu$, independent of the vector $\left(N, B, A_{1}, A_{2}, \ldots\right)$. Then any fixed point of $\widetilde{T}$ is characterized as a distribution of a random variable $X$ that satisfies (1.2).

This equation has gained importance in the last few years, since it turns out to be closely related to important objects in the computer science: the Quicksort algorithm [21, 24] (and other divide and conquer algorithms), the Pagerank algorithm [26, 17, 18] (being in the heart of the Google engine) and in stochastic geometry [22]. The inhomogeneous equation was recently used to describe equilibrium distribution of a class of kinetic models see e.g. [7]

Equation (1.2) is also a generalization of the homogeneous smoothing transform, which is defined exactly as above but without the inhomogeneous term $B$, i.e. with $B=0$ a.s. Thus, we say that $X$ is a solution (or a fixed point) of a homogeneous smoothing transform if

$$
X \stackrel{d}{=} \sum_{i=1}^{N} A_{i} X_{i},
$$

where $X_{1}, X_{2}, \ldots$ are independent copies of $X$ and the vector $\left(N, A_{1}, A_{2}, ..\right)$ is independent of the sequence $\left\{X_{i}\right\}$. The last equation appeared in the literature already in the eighties in connection with studying interacting particle system [13]. It turned out also to have a number of applications e.g. in branching random walks [16].

Existence of solutions of (1.3) and their properties were deeply studied in [13, 20] (see also the recent paper [3]) and it turns out that their properties are encoded in the function

$$
m(t)=\mathbb{E}\left[\sum_{i=1}^{N} A_{i}^{t}\right] .
$$

Notice, equation (1.3) does not have a unique solution since $t X$ for $t \in \mathbb{R}$ also solves it as long as $X$ does. We summarize known results in the following Lemma

Lemma 1.5 ([13, 20]). If $\mathbb{E} N>1$ and $\inf _{s \in[0,1]} m(s) \leq 1$, then the set of solutions of $(1.3)$ is nonempty. Moreover if $\mathbb{E}\left[\left(\sum_{i=1}^{N} A_{i}\right)^{1+\delta}\right]<\infty, \mathbb{E}\left[N^{1+\delta}\right]<\infty$ and for some $\alpha \in(0,1)$ we have $m(\alpha)=1, m^{\prime}(\alpha) \leq 0$ then

$$
\begin{aligned}
& \lim _{x \rightarrow \infty} \mathbb{P}[X>x] x^{\alpha}=c \quad \text { if } m^{\prime}(\alpha)<0, \\
& \lim _{x \rightarrow \infty} \mathbb{P}[X>x] x^{\alpha} / \log x=c \quad \text { if } m^{\prime}(\alpha)=0,
\end{aligned}
$$

for some positive constant $c$.

We begin the study of the nonhomogeneous smoothing transform explaining how to construct a solution to equation (1.2) (see [5,4] for more details). Let $\mathcal{T}=\bigcup_{k \geq 0} \mathbb{N}^{k}$ be an infinite Ulam-Harris tree, where $\mathbb{N}^{0}=\{\emptyset\}$. For $v=\left(i_{1}, \ldots, i_{n}\right)$ we define the length $|v|=n$ and by $v i$ we denote the vertex $\left(i_{1}, i_{2}, \ldots, i_{n}, i\right)$. We write $u<v$ if $u$ is a proper prefix of $v$, i.e. $u=\left(i_{1}, . ., i_{k}\right)$ for some $k<n$. Moreover we write $u \leq v$ if $u<v$ or $u=v$. Now we take $\left\{\left(B(v), A_{1}(v), A_{2}(v), \ldots\right)\right\}_{v \in \mathcal{T}}$ a family of i.i.d. copies of $\left(B, A_{1}, A_{2}, \ldots\right)$ indexed by the vertices of $\mathcal{T}$. For $v \in \mathcal{T}$ we also define a random variable $L(\emptyset)=1$ and $L(v i)=L(v) A_{i}(v)$. We can define now

$$
R=\sum_{v \in \mathcal{T}} L(v) B(v) .
$$

One can easily check that if the series above is finite almost surely then the random variable $R$ satisfies (1.2). However also the converse is true. Alsmeyer and Meiners [5] proved that existence 
of solutions of (1.2) is equivalent to finiteness of the series (1.6). Knowing that there exists one solution, one can construct a whole family of solutions just adding to $R$ any $Y$ being a solution of (1.3). However $R$ is distinguished by the property that it is the minimal solution (in the sense of stochastic domination i.e. $\mathbb{P}[R>t] \leq \mathbb{P}[X>t]$ for any other solution $X$ ), see [5] for more details. Another useful property is that $R$ is the unique solution that is measurable with respect to the input data $\left(B(v), A_{1}(v), A_{2}(v), \ldots\right)_{v \in \mathcal{T}}$ (compare with [2] where it is called an endogeneous solution). From now we call $R$ the minimal solution. Therefore, if we can describe the tail of $R$, in view Lemma 1.5, we obtain a full description of tails of all solutions (1.2).

Similarly like in the homogeneous case the fundamental role in description of solutions of (1.2) plays the function $m$ defined in (1.4). The necessary condition ensuring finiteness of (1.6) is that $m\left(t_{0}\right) \leq 1$ for some $t_{0} \in[0,1]$. However sufficient conditions are still not established. It is known $[5,17]$ that if $m(s)<1$ for some $s \in(0,1)$ and $\mathbb{E} B^{s}<\infty$ then $R$ is well defined.

Jelenković and Olvera-Cravioto $[17,18]$ proved that $R$ has a power law distribution:

Lemma 1.7. Let $\left(B, A_{1}, A_{2}, \ldots\right)$ be a nonnegative random vector, with $N \in \mathbb{N} \cup\{\infty\}, \mathbb{P}[B>0]>0$ and $R$ be the minimal solution to (1.2) given by (1.6). Suppose that

- the equation $m(s)=1$ has 2 solutions $\alpha<\beta$;

- $\mathbb{E}\left[B^{\beta}\right]<\infty, 0<m^{\prime}(\beta)=\mathbb{E}\left[\sum_{i=1}^{N} A_{i}^{\beta} \log A_{i}\right]<\infty$.

- there exists $j \geq 1$ with $\mathbb{P}\left[N \geq j, A_{j}>0\right]>0$ such that the measure $\mathbb{P}\left[\log A_{j} \in d u, A_{j}>0, N \geq j\right]$ is nonarithmetic;

In addition, assume that

a) $m(1)=\mathbb{E}\left[\sum_{i=1}^{N} A_{i}\right]<1$ and $\mathbb{E}\left[\left(\sum_{i=1}^{N} A_{i}\right)^{\beta}\right]<\infty$, if $\beta>1$;

or

b) $\mathbb{E}\left[\left(\sum_{i=1}^{N} A_{i}^{\beta /(1+\varepsilon)}\right)^{1+\varepsilon}\right]<0$ for some $0<\varepsilon<1$, if $0<\beta \leq 1$.

Then,

for some $C>0$.

$$
\mathbb{P}[R>t] \sim C t^{-\beta}, \quad t \rightarrow \infty,
$$

Remark 1.8. Positivity of the constant $C$ was not discussed in $[17,18]$ and was proved recently in [12].

Summarizing if $\alpha<\beta$ are two solutions of the equation $m(s)=1$ and $\alpha<1$, then the minimal solution $R$ of (1.2) has a power law of index $\beta$. Any other solution $X$ of $(1.2)$ satisfies $\mathbb{P}[X>t] \sim$ $C t^{-\alpha}$.

The main purpose of the present paper is to complete the picture and to study the critical case, when the equation $m(s)=1$ has exactly one solution $\alpha<1$ and then $m^{\prime}(\alpha)=0$, i.e. when the graph of the function $m(s)$ is tangent to the line $y=1$. For the random difference equation (1.1) this corresponds to the situation when the graph of the Mellin transform $\mathbb{E}\left[A^{s}\right]$ is tangent to the line $y=1$ at 0 , i.e. when $\mathbb{E}[\log A]=0$. Then it is known that equation (1.1) has no solutions, nevertheless when written in terms of measures has solutions in the class of Radon measures on $\mathbb{R}$. Existence and asymptotic properties of solutions were studied in $[6,8,9]$. For the homogeneous smoothing transform the critical case was considered by Durrett, Liggett [13] and Liu [20] and is a part of Lemma 1.5 (see also [10] for the case $\alpha=1$ ).

The only result we know concerning the inhomogeneous smoothing transform in the critical case is due to Alsmeyer and Meiners [5], who proved that for $\alpha<1 / 5$ (and under some further assumptions) the series (1.6) is finite, providing thus a solution to (1.2).

The main result of this paper is the following 
Theorem 1.9. Suppose that

$$
\begin{aligned}
& \text { There exists } 0<\alpha<1 \text { such that } m(\alpha)=\mathbb{E}\left[\sum_{i=1}^{N} A_{i}^{\alpha}\right]=1, \\
& m^{\prime}(\alpha)=\mathbb{E}\left[\sum_{i=1}^{N} A_{i}^{\alpha} \log A_{i}\right]=0, \\
& \mathbb{E}[N]>1, \\
& \text { For some } j \text { the measure } \mathbb{P}\left[\log A_{j} \in d u, A_{j}>0, N \geq j\right] \text { is nonarithmetic, } \\
& \mathbb{E}\left[N^{1+\delta}+B^{\alpha+\delta}+\sum_{i=1}^{N}\left(A_{i}^{-\delta}+A_{i}^{\alpha+\delta}\right)\right]<\infty, \text { for some } 0<\delta<1-\alpha .
\end{aligned}
$$

Then the minimal solution $R$ of (1.2) is well defined and moreover

$$
\lim _{t \rightarrow \infty} t^{\alpha} \mathbb{P}[R>t]=C_{+}
$$

and the constant $C_{+}$is strictly positive.

Thus in the critical case the tail of the minimal solution of (1.2) is of the order $t^{-\alpha}$, whereas the tails of all the other solutions behaves at infinity like $\log t t^{-\alpha}$.

We finish the introduction with an overview over the organization of the paper. In Section 2 we prove that $\mathbb{P}[R>t] \leq C t^{-\alpha}$, that in particular implies that $R$ is finite a.s. The most essential part of the proof is contained in Section 3. We reduce the problem to study behavior at 0 of the Laplace transform $\phi$ of $R$. Considering $\phi$ as a solution of the Poisson equation we first prove that it behaves regularly at 0 (Section 3.1) and the deduce the correct asymptotic (Section 3.3). Finally, applying some arguments based on the Landau theorem and holomorphic functions, we prove positivity of the limiting constant (Section 3.4).

The authors are grateful to Jacek Zienkiewicz for stimulating discussions on the subject of the paper.

\section{EXISTENCE OF A SOlUtion AND its First ESTimates}

In this section we prove

Proposition 2.1. Assume hypotheses of Theorem 1.9 are satisfied, then

$$
\mathbb{P}[R>t] \leq C t^{-\alpha},
$$

where $R$ is the random variable defined in (1.6). In particular $R$ is finite a.s.

Corollary 2.2. $\mathbb{E}\left[R^{\beta}\right]$ is finite for every $\beta<\alpha$.

We start with the following lemma

Lemma 2.3. Let $\left\{Y_{n}\right\}_{n \in \mathbb{N}}$ be a sequence of i.i.d. random variables such that $\mathbb{E} Y_{1}=0$. Let $S_{n}=$ $\sum_{i=1}^{n} Y_{i}$ be the sequence of the partial sums. Then, for any strictly positive constant $\delta$, the function

$$
\left.W(x)=\mathbb{E}\left[\sum_{i=0}^{\infty} e^{-\delta\left(x+S_{i}\right)} \mathbf{1}_{\left[S_{j}+x \geq 0\right.} \text { for } j \leq i\right]\right],
$$

is bounded. 
Proof. By definition the function $W$ can be nonzero only for positive $x$. Let $L=\inf \left\{i: S_{i}<0\right\}$, then

$$
\begin{aligned}
W(x) & =\mathbb{E}\left[\sum_{i=0}^{\infty} e^{-\delta\left(x+S_{i}\right)} \mathbf{1}_{\left[S_{j}+x \geq 0 \text { for } j \leq i\right]}\right] \\
& =\mathbb{E}\left[\sum_{i=0}^{L-1} e^{-\delta\left(x+S_{i}\right)} \mathbf{1}_{\left[S_{j}+x \geq 0 \text { for } j \leq i\right]}\right]+\mathbb{E}\left[\sum_{i=L}^{\infty} e^{-\delta\left(x+S_{i}\right)} \mathbf{1}_{\left[S_{j}+x \geq 0 \text { for } j \leq i\right]}\right] \\
& =\mathbb{E}\left[\sum_{i=0}^{L-1} e^{-\delta\left(x+S_{i}\right)}\right] \mathbf{1}_{[x \geq 0]}+\mathbb{E}\left[\sum_{i=L}^{\infty} e^{-\delta\left(x+S_{i}\right)} \mathbf{1}_{\left[S_{j}+x \geq 0 \text { for } L \leq j \leq i\right]}\right] \\
& =\mathbb{E}\left[\sum_{i=0}^{L-1} e^{-\delta S_{i}}\right] e^{-\delta x} \mathbf{1}_{[x \geq 0]}+\mathbb{E}\left[W\left(x+S_{L}\right)\right]
\end{aligned}
$$

Notice that the first expression above is just a finite constant, since by the reflection principle [14]

$$
\mathbb{E}\left[\sum_{i=0}^{L-1} e^{-\delta S_{i}}\right]=\mathbb{E}\left[\sum_{n=0}^{\infty} e^{-\delta S_{T_{n}}}\right]=: C<\infty,
$$

where $T_{n}$ is the sequence of upward ladder times: $T_{0}=0, T_{n}=\inf \left\{i>T_{n-1}: S_{i} \geq S_{T_{n-1}}\right\}$. Moreover the function $f(x)=e^{-\delta x} \mathbf{1}_{[x \geq 0]}$ is directly Riemann integrable (dRi), i.e. it is integrable and satisfies

$$
\lim _{h \rightarrow 0} h \sum_{n \in \mathbb{Z}} \sup _{x, y \in I_{n}(h)}|f(x)-f(y)|=0,
$$

where $I_{n}(h)=(n h,(n+1) h]$.

Finally, we can express $W$ as the convolution of the function $f$ with the potential of the transient random walk $V_{n}$, where $V_{n}$ is the sum of $n$ independent copies of $S_{L}$. Therefore, independently of $x$, we have

$$
\begin{aligned}
W(x) & =\sum_{n=0}^{\infty} f\left(x+V_{n}\right) \leq C \sum_{n=0}^{\infty} \sum_{k=0}^{\infty} e^{-\delta k} \mathbf{1}_{\left[x+V_{n} \in[k, k+1)\right]} \\
& \leq C \sum_{k=0}^{\infty} e^{-\delta k} \sum_{n=0}^{\infty} \mathbb{P}\left[x-k+V_{n} \in[0,1)\right] \leq C^{\prime} \sum_{k=0}^{\infty} e^{-\delta k}<\infty,
\end{aligned}
$$

where the uniform bound in the last line follows from Proposition 2.1 in [23, CH. 5].

Let us introduce a random variable $Y$ with distribution given by

$$
\mathbb{E}[f(Y)]=\mathbb{E}\left[\sum_{i=1}^{N} f\left(-\log A_{i}\right) A_{i}^{\alpha}\right],
$$

for any positive Borel function $f$. By (1.10), the right hand side indeed defines a probability measure. The main properties of $Y$, we are going to use, are summarized in the following lemma

Lemma 2.6. The random variable $Y$ is centered $(\mathbb{E} Y=0$ ), nonarithmetic (the closed subgroup generated by the support of the measure $\mathbb{P}[Y \in d x]$ is $\mathbb{R}$ ) and has finite exponential moments

$$
\mathbb{E}\left[e^{ \pm \delta Y}\right]<\infty
$$

for some $\delta>0$. 
Proof. We have

$$
\begin{aligned}
& \mathbb{E}\left[e^{ \pm \delta Y}\right]=\mathbb{E}\left[\sum_{i=1}^{N} A_{i}^{\alpha \mp \delta}\right]=m(\alpha \mp \delta)<\infty, \\
& \mathbb{E}[Y]=\mathbb{E}\left[\sum_{i=1}^{N}-A_{i}^{\alpha} \log A_{i}\right]=0 .
\end{aligned}
$$

Nonarithmecity follows from assumption (1.13).

Proof of Proposition (2.1). We compare behavior of the sum $R=\sum_{v \in \mathcal{T}} L(v) B(v)$ with behavior of the maximum $\widetilde{R}=\max _{v \in \mathcal{T}} L(v)$.

We first prove that

$$
\mathbb{P}[\widetilde{R}>t] \leq C t^{-\alpha},
$$

for some positive constant $C$.

Let $\left\{Y_{i}\right\}$ be a sequence of independent copies of $Y$ defined in (2.5) and let $S_{n}$ be the sequence of their partial sums. Applying the definition of $Y$ and reasoning by the induction (see e.g. [1]) one can easily prove the following well-known formula:

$$
\mathbb{E}\left[e^{\alpha S_{n}} f\left(S_{1}, \ldots, S_{n}\right)\right]=\mathbb{E}\left[\sum_{|v|=n} f\left(-\log L\left(v_{1}\right), \ldots,-\log L\left(v_{n}\right)\right)\right],
$$

valid for a fixed $n$ and any test function $f: \mathbb{R}^{n} \rightarrow \mathbb{R}$.

Putting $f\left(x_{1}, . ., x_{n}\right)=\mathbf{1}_{\left[x_{1} \geq-\log t, \ldots, x_{n-1} \geq-\log t, x_{n}<-\log t\right]}$ we obtain

$$
\begin{aligned}
\mathbb{P}[\widetilde{R}>t] & =\mathbb{P}[L(v)>t, \text { for some } v \in \mathcal{T}] \\
& =\sum_{n} \mathbb{P}[L(v)>t \text { for some }|v|=n \text { and } L(u) \leq t \text { for } u \leq v] \\
& \leq \sum_{n} \mathbb{E}\left[\sum_{|v|=n} \mathbf{1}_{\left[-\log L\left(v_{1}\right) \geq-\log t, \ldots,-\log L\left(v_{n-1}\right) \geq-\log t,-\log L(v)<-\log t\right]}\right] \\
& =\sum_{n} \mathbb{E}\left[e^{\alpha S_{n}} \mathbf{1}_{\left[S_{1} \geq-\log t, \ldots, S_{n-1} \geq-\log t, S_{n}<-\log t\right]}\right] \\
& \leq t^{-\alpha},
\end{aligned}
$$

hence we obtain (2.7).

Next we write

$$
\mathbb{P}[R>t] \leq \mathbb{P}[\widetilde{R}>t]+\mathbb{P}[\{R>t\} \cap\{\widetilde{R} \leq t\}] .
$$

In view of (2.7) it is sufficient to estimate only the second term. Taking $\gamma=\alpha+\delta<1$, we have

$$
\begin{aligned}
\mathbb{P}[\{R>t\} \cap\{\widetilde{R} \leq t\}] & \leq \mathbb{P}\left[\sum_{v \in \mathcal{T}} L(v) B(v) \mathbf{1}_{\left[L\left(v^{\prime}\right) \leq t \text { for } v^{\prime} \leq v\right]}>t\right] \\
& \leq \mathbb{P}\left[\sum_{v \in \mathcal{T}} L(v)^{\gamma} B(v)^{\gamma} \mathbf{1}_{\left[L\left(v^{\prime}\right) \leq t \text { for } v^{\prime} \leq v\right]}>t^{\gamma}\right] \\
& \leq \frac{1}{t^{\gamma}} \mathbb{E}\left[B^{\gamma}\right] \mathbb{E}\left[\sum_{v \in \mathcal{T}} L(v)^{\gamma} \mathbf{1}_{\left[L\left(v^{\prime}\right) \leq t \text { for } v^{\prime} \leq v\right]}\right]
\end{aligned}
$$


Applying again (2.8) we obtain

$$
\begin{aligned}
\mathbb{E}\left[\sum_{v \in \mathcal{T}} L(v)^{\gamma} \mathbf{1}_{\left[L\left(v^{\prime}\right) \leq t \text { for } v^{\prime} \leq v\right]}\right] & =\sum_{n} \mathbb{E}\left[\sum_{|v|=n} L(v)^{\gamma} \mathbf{1}_{\left[L\left(v^{\prime}\right) \leq t \text { for } v^{\prime} \leq v\right]}\right] \\
& =\sum_{n} \mathbb{E}\left[e^{\alpha S_{n}} e^{-\gamma S_{n}} \mathbf{1}_{\left[S_{k}+\log t \geq 0 \text { for } k \leq n\right]}\right] \\
& =\sum_{n} \mathbb{E}\left[e^{-(\delta)\left(S_{n}+\log t\right)} t^{\delta} \mathbf{1}_{\left[S_{k}+\log t \geq 0 \text { for } k \leq n\right]}\right] \\
& =t^{\delta} W(\log t),
\end{aligned}
$$

where $W$ is the function defined in Lemma 2.3, which as we already know is bounded. Finally, since $\mathbb{E}\left[B^{\gamma}\right]<\infty$, we obtain

$$
\mathbb{P}[\{R>t\} \cap\{\widetilde{R} \leq t\}] \leq C t^{-\alpha}
$$

\section{TAIL OF THE SOLUTION}

3.1. The Poisson equation. For a non-negative random variable $X$ by $\phi_{X}(t)=\mathbb{E}\left[e^{-t X}\right]$ we denote its Laplace transform. For simplicity we write $\phi=\phi_{R}$ for the Laplace transform of $R$. To prove our main result we use the duality between the tail behaviour of $R$ and the behaviour of its Laplace transform $\phi$ near 0 given by the following Tauberian theorem (its proof can be found e.g. in the book of Feller [14], Example c) after Theorem 4 in Chap. XIII).

Lemma 3.1 (Tauberian Theorem). For $0<\alpha<1$ and a slowly varying function $L$ the following are equivalent:

$$
\begin{aligned}
\text { i) } & \lim _{x \rightarrow \infty} \frac{x^{\alpha} \mathbb{P}[R>x]}{L(x)}=1 \\
\text { ii) } & \lim _{t \rightarrow 0} \frac{1-\phi(t)}{t^{\alpha} L(1 / t)}=\Gamma(1-\alpha) .
\end{aligned}
$$

Thus, in order to describe the tail of $R$, i.e. $\mathbb{P}[R>t]$, it is sufficient to study its Laplace transform $\phi$ and prove

$$
\lim _{t \rightarrow 0} \frac{1-\phi(t)}{t^{\alpha}}=C
$$

It is convenient for our purpose to change the coordinates and define

$$
D(x)=e^{\alpha x}\left(1-\phi\left(e^{-x}\right)\right) .
$$

Then our aim is to prove

$$
\lim _{x \rightarrow \infty} D(x)=C .
$$

We will often use the following well-known lemma

Lemma 3.3. For any positive random variable $X$ and any $0<\gamma<1$ we have

$$
1-\phi_{X}(t) \leq \Gamma(1-\gamma) \mathbb{E}\left[X^{\gamma}\right] t^{\gamma} .
$$


Proof. Notice that $\mathbb{E}[f(X)]=\int_{0}^{\infty} f^{\prime}(s) \mathbb{P}[X>s] d s$ for nonnegative $X$ and any differentiable, monotone function $f$ such that $f(0)=0$. Then by Chebyshev inequality

$$
\begin{aligned}
1-\phi_{X}(t) & =\mathbb{E}\left[1-e^{-t X}\right]=\int_{0}^{\infty}\left(1-e^{-s}\right)^{\prime} \mathbb{P}[t X>s] d s \\
& \leq \int_{0}^{\infty} e^{-s} \mathbb{E}\left[(t X)^{\gamma}\right] s^{-\gamma} d s=\Gamma(1-\gamma) \mathbb{E}\left[X^{\gamma}\right] t^{\gamma} .
\end{aligned}
$$

To prove (3.2) we apply some techniques described in the paper of Durrett and Liggett [13], who considered solutions of the homogeneous equation (1.3). We adopt their ideas, however it turns out that adding the additional term $B$ causes serious problems, hence we will present here all the details of the proof.

Let $Y$ be the random variable defined in (2.5). We consider $D$ as a solution of the Poisson equation.

Lemma 3.4. The function D satisfies the following Poisson equation

$$
\mathbb{E}[D(x+Y)]=D(x)+G(x),
$$

where

$$
G(x)=e^{\alpha x} \mathbb{E}\left[\sum_{i=1}^{N}\left(1-\phi\left(e^{-x} A_{i}\right)\right)-\left(1-e^{-e^{-x} B} \prod_{i=1}^{N} \phi\left(e^{-x} A_{i}\right)\right)\right] .
$$

Proof. Notice first that rewriting equation (1.2) in terms of Laplace transform $\phi$ we obtain

$$
\phi(t)=\mathbb{E}\left[\prod_{i=1}^{N} \phi\left(t A_{i}\right) e^{-t B}\right] .
$$

Hence by the definition of $D$ and the equation above we have

$$
\begin{aligned}
\mathbb{E}[D(x+Y)]-D(x)=\mathbb{E}\left[e^{\alpha(x+Y)}\left(1-\phi\left(e^{-(x+Y)}\right)\right)\right]-e^{\alpha x}\left(1-\phi\left(e^{-x}\right)\right) \\
=e^{\alpha x} \mathbb{E}\left[\sum_{i=1}^{N} e^{-\alpha \log A_{i}}\left(1-\phi\left(e^{-x+\log A_{i}}\right)\right) A_{i}^{\alpha}-\left(1-e^{-e^{-x} B} \prod_{i=1}^{N} \phi\left(e^{-x} A_{i}\right)\right)\right] \\
=e^{\alpha x} \mathbb{E}\left[\sum_{i=1}^{N}\left(1-\phi\left(e^{-x} A_{i}\right)\right)-\left(1-e^{-e^{-x} B} \prod_{i=1}^{N} \phi\left(e^{-x} A_{i}\right)\right)\right]=G(x)
\end{aligned}
$$

We need also the following technical lemma saying that for any $t \in(0, \alpha+\delta)$ the sum $\sum_{i=1}^{N} A_{i}^{t}$, that appears under the expected value in the definition of $m(1.4)$, has moment bigger than 1 .

Lemma 3.5. Assume that $\mathbb{E}\left[N^{1+\delta}\right]<\infty$ for some $\delta>0$. Let $X_{i}$ be an arbitrary sequence of random variables. Then for any $r>1$ and any $p \in\left(1, \frac{r(1+\delta)}{r+\delta}\right)$ we have

$$
\mathbb{E}\left[\left(\sum_{i=1}^{N} X_{i}^{1 / r}\right)^{p}\right] \leq C_{r, p} \mathbb{E}\left[\sum_{i=1}^{N} X_{i}\right]
$$

where $C_{r, p}$ is a constant depending on $r$ and $p$. 
Proof. We will use the following well-known inequality (being just a simple consequence of the Hölder inequality)

$$
\left(\sum_{i=1}^{n} a_{i}\right)^{r} \leq n^{r-1} \sum_{i=1}^{n} a_{i}^{r}
$$

where $a_{i} \geq 0$.

Applying first the last inequality and then the Hölder inequality with parameters $r / p$ and $(r / p)^{\prime}$ (given $q>1$, we denote by $q^{\prime}$ the conjugate real number such that $1 / q+1 / q^{\prime}=1$ ) we obtain

$$
\mathbb{E}\left[\left(\sum_{i=1}^{N} X_{i}^{1 / r}\right)^{p}\right] \leq \mathbb{E}\left[N^{(r-1) p / r}\left(\sum_{i=1}^{N} X_{i}\right)^{p / r}\right] \leq \mathbb{E}\left[N^{\frac{(r-1) p}{r}\left(\frac{r}{p}\right)^{\prime}}\right]^{1 /(r / p)^{\prime}} \mathbb{E}\left[\sum_{i=1}^{N} X_{i}\right]^{p / r} .
$$

Notice that since

$$
(r-1) \frac{p}{r}\left(\frac{r}{p}\right)^{\prime} \leq 1+\delta
$$

in view of our assumptions both expressions above are finite.

We prove now a weaker result than (3.2) saying that the function $D$ behaves regularly at infinity.

Proposition 3.6. Assume that hypotheses of Theorem 1.9 are satisfied. Then for any $y \in \mathbb{R}$ we have

$$
\lim _{x \rightarrow \infty} \frac{D(x+y)}{D(x)}=1 .
$$

Proof. We divide the proof of the proposition into several steps. Assume first additionally that the following condition is satisfied

$$
\lim _{t \rightarrow 0} \frac{1-\mathbb{E}\left[e^{-t B}\right]}{1-\phi(t)}=0
$$

Step 1. First we will show that

$$
\lim _{x \rightarrow \infty} \frac{G(x)}{D(x)}=0
$$

We can write

$$
\begin{aligned}
\lim _{x \rightarrow \infty} \frac{G(x)}{D(x)} & =\lim _{x \rightarrow \infty} \mathbb{E}\left[\frac{\prod_{i=1}^{N} \phi\left(e^{-x} A_{i}\right)\left(e^{-e^{-x} B}-1\right)}{1-\phi\left(e^{-x}\right)}\right] \\
& +\lim _{x \rightarrow \infty} \mathbb{E}\left[\frac{\prod_{i=1}^{N} \phi\left(e^{-x} A_{i}\right)-1+\sum_{i=1}^{N}\left(1-\phi\left(e^{-x} A_{i}\right)\right)}{1-\phi\left(e^{-x}\right)}\right]
\end{aligned}
$$

By our assumptions the first term is equal to 0 since the Laplace transform $\phi$ is bounded by 1 .

In order to show that the second limit (3.9) is zero we will use the following inequality valid for $0 \leq u_{i} \leq v_{i} \leq 1$

$$
\prod_{i=1}^{N} u_{i}-1+\sum_{i=1}^{N}\left(1-u_{i}\right) \geq \prod_{i=1}^{N} v_{i}-1+\sum_{i=1}^{N}\left(1-v_{i}\right) \quad(\text { see }[13,(2.5)])
$$

Next we will deduce that the expression under the expectation in (3.9) is positive. 
In order to bound this limit from above we use the inequality $u \leq e^{-(1-u)}$ for $u \in \mathbb{R}$. Therefore, we can write

$$
\begin{aligned}
& \mathbb{E}\left[\prod_{i=1}^{N} \phi\left(e^{-x} A_{i}\right)-1+\sum_{i=1}^{N}\left(1-\phi\left(e^{-x} A_{i}\right)\right)\right] \\
& \quad \leq \mathbb{E}\left[\exp \left(-\sum_{i=1}^{N}\left(1-\phi\left(e^{-x} A_{i}\right)\right)\right)-1+\sum_{i=1}^{N}\left(1-\phi\left(e^{-x} A_{i}\right)\right)\right] .
\end{aligned}
$$

Now we will split the problem into two separate cases

Step 1, case i) There exists constant $M$ such that for any $i, A_{i} \leq M$ a.s. Since $\phi$ is a Laplace transform of a non-negative random variable, $(1-\phi(u)) / u$ is a decreasing function whereas $(1-\phi(u))$ is increasing. Hence

$$
1-\phi\left(e^{-x} A_{i}\right) \leq \max \left(A_{i}, 1\right)\left(1-\phi\left(e^{-x}\right)\right)
$$

and thus

$$
\sum_{i=1}^{N}\left[1-\phi\left(e^{-x} A_{i}\right)\right] \leq\left(N+\sum_{i=1}^{N} A_{i}\right)\left(1-\phi\left(e^{-x}\right)\right) .
$$

Therefore, since the function $F(u)=e^{-u}-1+u$ is increasing on $[0, \infty), F(u) / u$ is bounded and tends to 0 as $u \rightarrow 0$ we can apply the Lebesgue theorem and obtain

$$
\begin{aligned}
& \limsup _{x \rightarrow \infty} \frac{\mathbb{E}\left[\prod_{i=1}^{N} \phi\left(e^{-x} A_{i}\right)-1+\sum_{i=1}^{N}\left(1-\phi\left(e^{-x} A_{i}\right)\right)\right]}{1-\phi\left(e^{-x}\right)} \\
& \leq \limsup _{x \rightarrow \infty} \frac{\mathbb{E}\left[F\left(\sum_{i=1}^{N}\left(1-\phi\left(e^{-x} A_{i}\right)\right)\right)\right]}{1-\phi\left(e^{-x}\right)} \leq \limsup _{t \rightarrow 0} \frac{\mathbb{E}\left[F\left(\left(N+\sum_{i=1}^{N} A_{i}\right) t\right)\right]}{t} \\
& \leq \limsup _{t \rightarrow 0} \mathbb{E}\left[\frac{F\left(\left(N+\sum_{i=1}^{N} A_{i}\right) t\right)}{\left(N+\sum_{i=1}^{N} A_{i}\right) t} \cdot\left(N+\sum_{i=1}^{N} A_{i}\right)\right]=0
\end{aligned}
$$

because $\mathbb{E}\left[\left(N+\sum_{i=1}^{N} A_{i}\right)\right]<(M+1) \mathbb{E}[N]<\infty$.

Step 1, case ii) $A_{i}$ 's are unbounded. Take $M>0$ big enough that will be specified later. Define a truncated random vector $\left(\widetilde{B}(v), \widetilde{A_{1}}(v), \ldots \widetilde{A_{N}}(v)\right)=\left(B(v) \wedge M, A_{1}(v) \wedge M, \ldots, A_{N}(v) \wedge M\right)$. Now take

$$
m_{M}(t)=\mathbb{E}\left[\sum_{i=1}^{N} \widetilde{A}_{i}^{t}\right]
$$

and observe that $m_{M}(t)=1$ has two different solution $\alpha_{M}<\alpha<\beta_{M}$ and both of them converge to $\alpha$ as $M \rightarrow \infty$. Indeed, it follows from the fact that $m_{M}(\alpha)<1$ and for $t \in(0, \alpha+\delta)$ different than $\alpha$ the Lebesgue Theorem gives that $m_{M}(t) \rightarrow m(t)>1$. We will assume that $\beta_{M}<\alpha+\delta$.

Define

$$
\widetilde{R}=\sum_{v \in \mathcal{T}} \widetilde{L}(v) \widetilde{B}(v),
$$

where $\widetilde{L}$ is defined in the same way as $L$ but in terms of $\widetilde{A_{i}}(v)$. Clearly $\widetilde{R} \leq R$ a.s. Since $M$ can be chosen in such a way that for any $j$ the measure $\mathbb{P}\left[\log \widetilde{A_{j}} \in d u, A_{j}>0, N \geq j\right]$ has the same 
support as $\mathbb{P}\left[\log A_{j} \in d u, A_{j}>0, N \geq j\right]$ we can apply Lemma 1.7 a) for the random variable $\widetilde{R}$ and obtain $\mathbb{P}[\widetilde{R}>t] \sim C_{+} t^{-\beta_{M}}$ for some positive $C_{+}$. Hence, by Tauberian Theorem

$$
1-\widetilde{\phi}(t) \sim C_{+} \Gamma\left(1-\beta_{M}\right) t^{\beta_{M}},
$$

where $\widetilde{\phi}(t)=\phi_{\widetilde{R}}(t) \geq \phi(t)$. Therefore we can find $C_{0}$ such that $1-\widetilde{\phi}(t) \geq C_{0} t^{\beta_{M}}$ for $0<t<1$. On the other hand, by Lemma 3.3 and Corollary 2.2,1- $\phi(t) \leq C_{1} t^{\beta}$ for $\beta<\alpha$. This implies that for some $C$ and any $t$

$$
1-\phi(t) \leq C(1-\widetilde{\phi}(t))^{\beta / \beta_{M}}
$$

From (3.12) get

$$
\lim _{t \rightarrow 0} \frac{1-\widetilde{\phi}(t s)}{1-\widetilde{\phi}(t)}=s^{\beta_{M}},
$$

hence for any $0<\varepsilon<\alpha \delta / 2$ we may find $C_{1}$ such that

$$
\frac{1-\widetilde{\phi}(s t)}{1-\widetilde{\phi}(t)} \leq C_{1} s^{\beta_{M}+\varepsilon},
$$

for any $t>0$ and $s>1$. Therefore, we have

$$
\begin{aligned}
1-\phi(t s) & \leq C(1-\widetilde{\phi}(t s)))^{\beta / \beta_{M}} \\
& \leq C\left(C_{1}(1-\widetilde{\phi}(t)) s^{\beta_{M}+\varepsilon}\right)^{\beta / \beta_{M}} \\
& \leq C_{2}(1-\widetilde{\phi}(t))^{\beta / \beta_{M}}\left(s^{\left(\beta_{M}+\varepsilon\right) \frac{\beta}{\beta_{M}}}\right) .
\end{aligned}
$$

Thus, since the function $F$ is increasing, $F(u) / u^{\frac{\beta_{M}}{\beta}}$ is bounded for $\beta_{M} \leq 2 \beta$ and $\phi(t) \leq \widetilde{\phi}(t)$ we have

$$
\begin{aligned}
\frac{F\left(\sum_{i=1}^{N}\left(1-\phi\left(e^{-x} A_{i}\right)\right)\right)}{1-\phi\left(e^{-x}\right)} & \leq \frac{F\left(\sum_{i=1}^{N}\left(1-\phi\left(e^{-x}\left(1 \vee A_{i}\right)\right)\right)\right)}{1-\phi\left(e^{-x}\right)} \\
& \leq \frac{F\left(\left(C_{2} \sum_{i=1}^{N}\left(1 \vee A_{i}^{\left(\beta_{M}+\varepsilon\right) \frac{\beta}{\beta_{M}}}\right)\right)\left(1-\widetilde{\phi}\left(e^{-x}\right)\right)^{\beta / \beta_{M}}\right)}{1-\widetilde{\phi}\left(e^{-x}\right)} \\
& \leq C\left(\sum_{i=1}^{N} 1 \vee A_{i}^{\left(\beta_{M}+\varepsilon\right) \frac{\beta}{\beta_{M}}}\right)^{\frac{\beta_{M}}{\beta}} \\
& \leq C\left(N^{\frac{\beta_{M}}{\beta}}+\left(\sum_{i=1}^{N} A_{i}^{\left(\beta_{M}+\varepsilon\right) \frac{\beta}{\beta_{M}}}\right)^{\frac{\beta_{M}}{\beta}}\right) .
\end{aligned}
$$

Now, applying Lemma 3.5 with $X_{i}=A_{i}^{\alpha}, r=\frac{(\alpha+\delta) \beta_{M}}{\beta\left(\beta_{M}+\varepsilon\right)}$ and $p=\frac{\beta_{M}}{\beta}$ (one can choose sufficiently large $M$ and $\beta_{M}$ close to $\beta$ such that the pair $r, p$ satisfies asumptions of Lemma 3.5) we obtain

$$
\mathbb{E}\left[\frac{F\left(\sum_{i=1}^{N}\left(1-\phi\left(e^{-x} A_{i}\right)\right)\right)}{1-\phi\left(e^{-x}\right)}\right] \leq C\left(\mathbb{E}\left[N^{1+\delta}\right]+\mathbb{E}\left[\sum_{i=1}^{N} A_{i}^{\alpha+\delta}\right]\right)
$$


Finally, by Lebesgue Theorem and since $F(u) / u \rightarrow 0$ as $u \rightarrow 0$ we deduce that

$$
\lim _{x \rightarrow \infty} \frac{\mathbb{E}\left[F\left(\sum_{i=1}^{N}\left(1-\phi\left(e^{-x} A_{i}\right)\right)\right)\right]}{1-\phi\left(e^{-x}\right)}=\mathbb{E}\left[\lim _{x \rightarrow \infty} \frac{F\left(\sum_{i=1}^{N}\left(1-\phi\left(e^{-x} A_{i}\right)\right)\right)}{1-\phi\left(e^{-x}\right)}\right]=0 .
$$

Step 2. Let us introduce a family of functions

$$
h_{x}(y)=\frac{D(x+y)}{D(x)} .
$$

Dividing the equation

$$
D(x+y)=\mathbb{E}[D(x+y+Y)]-G(x+y)
$$

by $D(x)$, we obtain

$$
h_{x}(y)=\mathbb{E}\left[h_{x}(y+Y)\right]-\frac{G(x+y)}{D(x+y)} h_{x}(y) .
$$

Since $D(y) e^{-\alpha y}=1-\phi\left(e^{-y}\right)$ is decreasing and $D(y) e^{(1-\alpha) y}=\left(1-\phi\left(e^{-y}\right)\right) / e^{-y}$ is increasing the same holds for functions $h_{x}$. Therefore we conclude that $h_{x}(y) \leq \max \left\{e^{\alpha y}, e^{(\alpha-1) y}\right\}$ and that $h_{x}$ are equi-continuous on bounded sets. By Arzelà-Ascoli theorem the set $\left\{h_{x}\right\}$ is relatively compact in the topology of uniform convergence on compact sets. Take now an accumulation point $h$ as $x \rightarrow \infty$ i.e. $h_{x_{n}} \rightarrow h$ for some sequence $x_{n} \rightarrow \infty$. Passing to infinity with $x_{n}$ in (3.13) from Step 1 and the Lebesgue theorem we have

$$
h(y)=\mathbb{E}[h(y+Y)] .
$$

Since any positive $Y$-harmonic function is constant it yields that $h(y)=h(0)=1$. Hence, $h$ is the unique accumulation point and therefore $D(x+y) / D(x) \rightarrow 1$.

Step 3. Finally we get rid of the additional assumption (3.7). We define $\tilde{B}=\min \{1, B\}$ and consider

$$
\widetilde{R}=\sum_{v \in \mathcal{T}} L(v) \tilde{B}(v)
$$

i.e. $\widetilde{R}$ is defined in the same way as $R$ in (1.2) but with $B(v)$ replaced by $\tilde{B}(v)$. Of course $\widetilde{R} \leq R$, hence $\widetilde{R}$ is also finite a.s. and solves the equation

$$
\tilde{X}={ }_{d} \sum_{i=1}^{N} A_{i} \tilde{X}_{i}+\tilde{B}
$$

with the vector $\left(\tilde{B}, A_{1}, A_{2}, \ldots\right)$ satisfying hypotheses (1.10)-(1.14).

Let $\tilde{\phi}=\phi_{\tilde{R}}$ be the Laplace transform of $\tilde{R}$. Notice that (3.7) is satisfied for $R$ and $B$ replaced by $\widetilde{R}$ and $\widetilde{B}$, i.e.

$$
\lim _{t \rightarrow 0} \frac{1-\mathbb{E}\left[e^{-t \widetilde{B}}\right]}{1-\widetilde{\phi}(t)}=0 .
$$

Indeed, observe first that $\mathbb{E}[\tilde{R}]=\infty$, otherwise we would have

$$
\mathbb{E}[\tilde{R}]=\mathbb{E}\left[\sum_{i=1}^{N} A_{i}\right] \mathbb{E}[\tilde{R}]+\mathbb{E}[\tilde{B}]
$$


which is impossible since $1<\mathbb{E}\left[\sum_{i=1}^{N} A_{i}\right] \leq \infty$. Thus in consequence $1-\tilde{\phi}(t) / t$ tends to infinity as $t$ goes to 0 . Moreover, since $\widetilde{B}$ is bounded a.s., by the Lebesgue theorem and by Lemma 3.3 we have

$$
\lim _{t \rightarrow 0} \frac{1-\mathbb{E}\left[e^{-t \tilde{B}}\right]}{1-\tilde{\phi}(t)}=\lim _{t \rightarrow 0} \mathbb{E}\left[\frac{1-e^{-t \tilde{B}}}{t}\right] \cdot \frac{t}{1-\tilde{\phi}(t)}=\mathbb{E}[\tilde{B}] \cdot \lim _{t \rightarrow 0} \frac{t}{1-\tilde{\phi}(t)}=0,
$$

which proves (3.15). Therefore, we may use the results proved in the first two steps of the proof saying that

$$
\lim _{x \rightarrow \infty} \frac{e^{\alpha(x+y)}\left(1-\widetilde{\phi}\left(e^{-(x+y)}\right)\right)}{e^{\alpha x}\left(1-\widetilde{\phi}\left(e^{-x}\right)\right)}=1
$$

Hence

$$
\frac{1-\tilde{\phi}(t)}{t^{\alpha}}=L(1 / t)
$$

for some slowly varying function $L$.

Since $\tilde{R} \leq R$ and $\tilde{\phi} \geq \phi$, for $0<\varepsilon<\delta$ we have

$$
\lim _{t \rightarrow 0} \frac{t^{\alpha+\varepsilon}}{1-\phi(t)} \leq \lim _{t \rightarrow 0} \frac{t^{\alpha+\varepsilon}}{1-\tilde{\phi}(t)}=\lim _{t \rightarrow 0} \frac{t^{\varepsilon}}{L(1 / t)}=0 .
$$

By Lemma 3.3

$$
\frac{1-\mathbb{E}\left[e^{-t B}\right]}{t^{\alpha+\varepsilon}} \leq C<\infty
$$

Finally, we get

$$
\lim _{t \rightarrow 0} \frac{1-\mathbb{E}\left[e^{-t B}\right]}{1-\phi(t)}=\lim _{t \rightarrow 0} \frac{1-\mathbb{E}\left[e^{-t B}\right]}{t^{\alpha+\varepsilon}} \cdot \frac{t^{\alpha+\varepsilon}}{1-\phi(t)}=0
$$

The last Proposition implies immediately the following results

Corollary 3.16. Under assumptions of Theorem 1.9 we have the following: for any $s>0$

$$
\lim _{t \rightarrow 0} \frac{1-\phi(t s)}{1-\phi(t)}=s^{\alpha} .
$$

In particular, the function $L(t)=(1-\phi(1 / t)) t^{\alpha}$ is slowly varying.

Corollary 3.18. Under assumptions (1.10)-(1.14) we have $\mathbb{E}\left[R^{\beta}\right]<\infty$ for $\beta<\alpha$ and $\mathbb{E}\left[R^{\beta}\right]=\infty$ for $\beta>\alpha$.

Proof. We have

$$
\mathbb{E}\left[R^{\beta}\right]=\beta \int_{0}^{\infty} t^{\beta-1} \mathbb{P}[R>t] d t=C_{0}+\beta \int_{1}^{\infty} t^{\beta-1-\alpha} L(t) d t
$$

what is finite if $\beta<\alpha$ and infinite if $\beta>\alpha$. 
3.2. Some properties of the function $G$. To prove our main results we will use the renewal theorem, therefore before we will proceed with the final arguments we have to prove some properties of the function $G$.

Lemma 3.19. There exists $\varepsilon>0$ such that the function $e^{\varepsilon|x|} G(x)$ is directly Riemman integrable.

Proof. For any $x$ and $\varepsilon<\min \{\delta, 1-\alpha\}$ we have

$$
\begin{aligned}
e^{ \pm \varepsilon x}|G(x)| \leq e^{(\alpha \pm \varepsilon) x} \mathbb{E}\left[\left|\sum_{i=1}^{N}\left(1-\phi\left(e^{-x} A_{i}\right)\right)-1+\prod_{i=1}^{N} \phi\left(e^{-x} A_{i}\right)\right|\right] \\
+e^{(\alpha \pm \varepsilon) x} \mathbb{E}\left[\left|\left(e^{-e^{-x} B}-1\right)\right| \prod_{i=1}^{N} \phi\left(e^{-x} A_{i}\right)\right] \\
=e^{(\alpha \pm \varepsilon) x} \mathbb{E}\left[\sum_{i=1}^{N}\left(1-\phi\left(e^{-x} A_{i}\right)\right)-1+\prod_{i=1}^{N} \phi\left(e^{-x} A_{i}\right)\right] \\
\quad+e^{(\alpha \pm \varepsilon) x} \mathbb{E}\left[\left(1-e^{-e^{-x} B}\right) \prod_{i=1}^{N} \phi\left(e^{-x} A_{i}\right)\right] \\
=f_{1}(x)+f_{2}(x),
\end{aligned}
$$

Notice first that since for $\gamma<\alpha+\varepsilon$, by Lemma (3.3) we have

$$
e^{\gamma x} \mathbb{E}\left[\left(1-e^{-e^{-x} B}\right) \prod_{i=1}^{N} \phi\left(e^{-x} A_{i}\right)\right] \leq e^{\gamma x}\left(1-\mathbb{E}\left[e^{-e^{-x} B}\right]\right) \leq C \min \left\{e^{\gamma x}, e^{(\gamma-\alpha-\delta) x}\right\},
$$

the function $f_{2}$ is directly Riemman integrable.

Let us now examine the function $f_{1}$. First, we will prove that $f_{1}$ is integrable. For this purpose notice that if $\gamma>0$ we have

$$
\begin{aligned}
\int_{\mathbb{R}} e^{\gamma x} \mathbb{E} & {\left[\sum_{i=1}^{N}\left(1-\phi\left(e^{-x} A_{i}\right)\right)-1+\prod_{i=1}^{N} \phi\left(e^{-x} A_{i}\right)\right] d x } \\
& \leq \int_{\mathbb{R}} e^{\gamma x} \mathbb{E}\left[\sum_{i=1}^{N}\left(1-\phi\left(e^{-x} A_{i}\right)\right)-1+e^{-\sum_{i=1}^{N}\left(1-\phi\left(e^{-x} A_{i}\right)\right)}\right] d x \\
& \leq \mathbb{E}\left[\int_{\mathbb{R}} e^{\gamma x} F\left(\sum_{i=1}^{N}\left(1-\phi\left(e^{-x} A_{i}\right)\right)\right) d x\right] .
\end{aligned}
$$

The above expression is finite for $\gamma<\alpha+\varepsilon$. Indeed, from the monotonicity of $F$ on the positive half line and from Lemma 3.3, for $\beta<\alpha$ we can bound the last integral by

$$
\begin{aligned}
\mathbb{E}\left[\int_{\mathbb{R}} e^{\gamma x} F\right. & \left.\left(C \sum_{i=1}^{N} A_{i}^{\beta} \mathbb{E}\left[R^{\beta}\right] e^{-\beta x}\right) d x\right] \\
= & \mathbb{E}\left[\left(C \mathbb{E}\left[R^{\beta}\right] \sum_{i=1}^{N} A_{i}^{\beta}\right)^{\gamma / \beta}\right] \times \int_{\mathbb{R}} e^{\gamma x} F\left(e^{-\beta x}\right) d x .
\end{aligned}
$$

To see that the expression above is finite we apply Lemma 3.5 with $r=(\alpha+\delta) / \alpha$ and $X_{i}=A_{i}^{r \beta}$. The second term is finite since $F(t) \leq \min \left\{t, t^{2} / 2\right\}$ and $\beta<\gamma<2 \beta$. Thus $f_{1}$ is integrable. 
Next we have to check that $f_{1}$ satisfies (2.4). Inequality (3.10) implies that $e^{-(\alpha \pm \varepsilon) x} f_{1}(x)$ is decreasing, hence also $e^{-x} f_{1}(x)$, since $\alpha+\varepsilon<1$. Therefore, for any $h>0$ and $x \in I_{n}(h)$ we have

$$
f_{1}(x) \leq e^{x-n h} f_{1}(n h) \leq e^{h} f_{1}(n h)
$$

and similarly we can estimate from below

$$
f_{1}(x) \geq e^{x-(n+1) h} f_{1}((n+1) h) \geq e^{-h} f_{1}((n+1) h) .
$$

Since $f_{1}$ is integrable, the series

$$
\sum_{n \in \mathbb{Z}} f_{1}(n h)=\sum_{n \in \mathbb{Z}} f_{1}((n+1) h) \leq \sum_{n \in \mathbb{Z}} \frac{1}{h} \int_{I_{n}(h)} e^{h} f_{1}(x) d x=\frac{e^{h}}{h} \int_{\mathbb{R}} f_{1}(x) d x<\infty
$$

is finite. We can write

$$
\begin{aligned}
\sum_{n \in \mathbb{Z}} \sup _{x, y \in I_{n}(h)} & \left|f_{1}(x)-f_{1}(y)\right| \cdot h \leq \sum_{n \in \mathbb{Z}}\left(e^{h} f_{1}(n h)-e^{-h} f_{1}((n+1) h)\right) \cdot h \\
& \leq \sum_{n \in \mathbb{Z}}\left(e^{h}-e^{-h}\right) f_{1}(n h) \cdot h \leq \sum_{n \in \mathbb{Z}}\left(e^{h}-e^{-h}\right) \int_{I_{n}(h)} f_{1}(x) e^{h} d x \\
& =\left(e^{2 h}-1\right) \int_{\mathbb{R}} f_{1}(x) d x .
\end{aligned}
$$

The last expression converges to 0 as $h$ goes to 0 , thus $f_{1}$ is directly Riemann integrable.

Corollary 3.20. Functions $x G(x)$ and $G(x)$ are directly Riemman integrable.

Proof. The corollary follows from the fact that $(1+|x|)|G(x)| \leq C\left(e^{-\varepsilon_{0} x}+e^{\varepsilon_{0} x}\right)|G(x)|$, for some sufficiently large $C$.

Corollary 3.21. If $\int G(x) d x=0$ then the function $\bar{G}(x)=\int_{-\infty}^{x} G(y) d y$ is also dRi and satisfies $\int \bar{G}(x) d x=-\int x G(x) d x$.

Proof. For $x \leq 0$ we have

$$
|\bar{G}(x)| \leq\left|\int_{-\infty}^{x} G(y) d y\right| \leq \int_{-\infty}^{x} e^{\varepsilon_{0} x} e^{-\varepsilon_{0} y}|G(y)| d y \leq C e^{\varepsilon_{0} x} .
$$

In the same way we prove for $x>0$ that

$$
|\bar{G}(x)| \leq\left|\int_{-\infty}^{x} G(y) d y\right|=\left|\int_{x}^{\infty} G(y) d y\right| \leq C e^{-\varepsilon_{0} x} .
$$

Hence $\bar{G}$ is directly Riemman integrable. Moreover,

$$
\begin{aligned}
\int_{\mathbb{R}} \bar{G}(x) d x & =\int_{\mathbb{R}} \int_{-\infty}^{x} G(y) d y d x \\
& =\int_{x \leq 0} \int_{-\infty}^{x} G(y) d y d x-\int_{x>0} \int_{x}^{\infty} G(y) d y d x \\
& =\int_{y \leq 0} \int_{y}^{0} G(y) d x d y-\int_{y>0} \int_{0}^{y} G(y) d x d y=-\int_{\mathbb{R}} y G(y) d y .
\end{aligned}
$$


3.3. Existence of the limit. We are able now to describe behaviour of $\phi$ near 0 .

Proposition 3.22. Under hypotheses (1.10)-(1.14) we have

$$
\lim _{t \rightarrow 0} \frac{1-\phi(t)}{t^{\alpha}}=C_{+}
$$

Thus, by the Tauberian theorem (Lemma 3.1) we deduce

Corollary 3.23. Under hypotheses (1.10)-(1.14) we have

$$
\lim _{x \rightarrow \infty} x^{\alpha} \mathbb{P}[R>x]=C_{+}
$$

Proof of Proposition 3.22. The scheme of the proof is similar to the proof of Theorem 2.18 in [13]. Let $\left\{Y_{n}\right\}$ be a sequence of independent copies of $Y$ defined in (2.5). By $S_{n}$ we denote their partial sums, i.e. $S_{n}=\sum_{i=1}^{n} Y_{i}$. We define the stopping time $L=\inf \left\{n \geq 0: S_{n}<0\right\}$ and the sequence of stopping times $T_{k}=\inf \left\{n>T_{k-1}: S_{n} \geq S_{T_{k-1}}\right\}$.

Since $D$ is a solution of the Poisson equation the sequence of random variables

$$
M_{n}(x)=D\left(x+S_{n}\right)-\sum_{i=0}^{n-1} G\left(x+S_{i}\right)
$$

forms a martingale with respect to the natural filtration generated by $\left\{Y_{n}\right\}$. In view of the optional stopping theorem we have

$$
\mathbb{E}\left[M_{n \wedge L}(x)\right]=\mathbb{E}\left[M_{0}(x)\right]=D(x)
$$

and equivalently

$$
\mathbb{E}\left[D\left(x+S_{n \wedge L}\right)\right]-D(x)=\mathbb{E}\left[\sum_{i=0}^{n \wedge L-1} G\left(x+S_{i}\right)\right] .
$$

Next we want to pass with $n$ to infinity. Notice that by the duality principle [14]

$$
\mathbb{E}\left[\sum_{i=0}^{L-1}\left|G\left(x+S_{i}\right)\right|\right]=\mathbb{E}\left[\sum_{i=0}^{\infty}\left|G\left(x+S_{T_{i}}\right)\right|\right]
$$

and the last sum is finite since $G$ is dRi, hence we can pass with $n \rightarrow \infty$ on the right side. In order to justify passing to limit on the left side observe that by Proposition 3.6 for any $\varepsilon<\delta$ we have $D(x) \leq C e^{\varepsilon|x|}$. Since $\mathbb{E}\left[e^{\varepsilon S_{L}}\right]<\infty$ (see [14] (3.6a) in Chap. XII), we can pass to infinity.

Thus we obtain

$$
\mathbb{E}\left[D\left(x+S_{L}\right)\right]-D(x)=\mathbb{E}\left[\sum_{i=0}^{L-1} G\left(x+S_{i}\right)\right]=: R(x) .
$$

Applying again the duality principle we have

$$
R(x)=\sum_{n=0}^{\infty} G\left(x+S_{T_{n}}\right) .
$$

Now the renewal theorem yields that

$$
\lim _{x \rightarrow \infty} R(x)=-\frac{\int_{\mathbb{R}} G(x) d x}{\mathbb{E}\left[S_{T_{1}}\right]} .
$$

Integrating (3.24) we have

$$
\int_{0}^{x}\left(\mathbb{E}\left[D\left(y+S_{L}\right)\right]-D(y)\right) d y=\int_{0}^{x} R(y) d y
$$


what can be rewritten as

$$
D(x) \cdot \mathbb{E}\left[\int_{0}^{S_{L}} \frac{D(x+y)}{D(x)} d y\right]-\mathbb{E}\left[\int_{0}^{S_{L}} D(y) d y\right]=\int_{0}^{x} R(y) d y
$$

By Proposition 3.6, $D(x+y) / D(x) \leq C e^{\varepsilon y}$ hence again the same argument as before tells us that we can pass to the limit under the integral sign and obtain

$$
\lim _{x \rightarrow \infty} \mathbb{E}\left[\int_{0}^{S_{L}} \frac{D(x+y)}{D(x)} d y\right]=\mathbb{E}\left[S_{L}\right]
$$

Dividing by $x$ in (3.28) and passing to the limit we obtain

$$
\lim _{x \rightarrow \infty} \frac{D(x)}{x} \mathbb{E}\left[S_{L}\right]=\lim _{x \rightarrow \infty} R(x)=-\frac{\int_{\mathbb{R}} G(x) d x}{\mathbb{E}\left[S_{T_{1}}\right]},
$$

and finally

$$
\lim _{x \rightarrow \infty} \frac{D(x)}{x}=-\frac{\int_{\mathbb{R}} G(x) d x}{\mathbb{E}\left[S_{T_{1}}\right] \mathbb{E}\left[S_{L}\right]}=\frac{2 \int_{\mathbb{R}} G(x) d x}{\sigma^{2}},
$$

where $\sigma^{2}:=\operatorname{Var} Y=2 \mathbb{E}\left[-S_{L}\right] \mathbb{E}\left[S_{T_{1}}\right]$ (see the proof of T18.1 on page 196 in [25]).

Notice that in view of Lemma 3.1 the last formula implies

$$
\lim _{t \rightarrow \infty} \frac{t^{\alpha}}{\log t} \mathbb{P}[R>t]=\frac{2 \int_{\mathbb{R}} G(x) d x}{\sigma^{2}} .
$$

By Proposition 2.1 the last constant must be 0 , therefore

$$
\int_{\mathbb{R}} G(x) d x=0 .
$$

Now we repeat the above procedure. Integrating (3.24) we have

$$
\int_{-\infty}^{x}\left(\mathbb{E}\left[D\left(y+S_{L}\right)\right]-D(y)\right) d y=\int_{-\infty}^{x} \mathbb{E}\left[\sum_{i=0}^{\infty} G\left(y+S_{T_{i}}\right)\right] d y
$$

what is equivalent to

$$
\mathbb{E}\left[\int_{0}^{S_{L}} D(x+y) d y\right]=\mathbb{E}\left[\sum_{i=0}^{\infty} \int_{-\infty}^{x} G\left(y+S_{T_{i}}\right) d y\right]=\mathbb{E}\left[\sum_{i=0}^{\infty} \bar{G}\left(x+S_{T_{i}}\right)\right] .
$$

Passing with $x$ to infinity in

$$
D(x) \mathbb{E}\left[\int_{0}^{S_{L}} \frac{D(x+y)}{D(x)} d y\right]=\mathbb{E}\left[\sum_{i=0}^{\infty} \bar{G}\left(x+S_{T_{i}}\right)\right]
$$

we obtain

$$
\lim _{x \rightarrow \infty} D(x)=\frac{2 \int \bar{G}(x) d x}{\sigma^{2}}=\frac{-2 \int x G(x) d x}{\sigma^{2}}
$$


3.4. Positivity of the limiting constant. Now we proceed with the last step of the proof and we justify that the constant $C_{+}$in the statement of Corollary 3.23 is strictly positive. We follow here the method developed in [11].

The key tool to deal with this problem is the Landau Theorem. Originally it was stated for Dirichlet series but it can be extended for functions of the type $f(s)=\int x^{s} \mu(d x)$ where $\mu$ is a positive measure on $\mathbb{R}^{+}$(compare with [27, Theorems 5a and 5b in Chap. II])

Theorem 3.30 (Landau). Let $\sigma_{c}$ be the abscissa of convergence for $f(z)=\int_{0}^{\infty} x^{z} \mu(d x)$, i.e. the integral converges for $\Re z<\sigma_{c}$ and diverges for $\Re z>\sigma_{c}$. Then $\sigma_{c}$ is a singularity for $f$.

As a conclusion we get that if $z \mapsto \mathbb{E}\left[R^{z}\right]$ has an analytic extension on some open neighborhood of $\alpha$ then $\mathbb{E}\left[R^{z}\right]$ is well defined there. Our aim is to find such an extension, under the assumptions that $\int G(x) d x=0$ and $\int x G(x) d x=0$.

Theorem 3.31. Under assumptions (1.10)-(1.14) the constant $C_{+}$is strictly positive.

Proof. Let us define

$$
H(z)=\int_{\mathbb{R}} e^{x z} \mathbb{E}\left[\sum_{i=1}^{N}\left(1-\phi\left(e^{-x} A_{i}\right)\right)-\left(1-\phi\left(e^{-x}\right)\right)\right] d x .
$$

By Lemma 3.19, $H$ is well defined for $0<\Re z<\alpha+\varepsilon_{0}$. Moreover, $H$ is a holomorphic function for $0<\Re z<\alpha+\varepsilon_{0}$. Indeed, take any closed piecewise $C^{1}$ curve $\gamma$ in $0<\Re z<\alpha+\varepsilon_{0}$. Then by Lemma 3.19 we have

$$
\begin{array}{r}
\int_{\gamma} \int_{\mathbb{R}}\left|e^{x z} \mathbb{E}\left[\sum_{i=1}^{N}\left(1-\phi\left(e^{-x} A_{i}\right)\right)-\left(1-\phi\left(e^{-x}\right)\right)\right]\right| d x|d z| \\
\leq \int_{\gamma}|d z| \int\left(e^{-\varepsilon_{0} x}+e^{\varepsilon_{0} x}\right)|G(x)| d x<\infty .
\end{array}
$$

Hence, by the Fubini Theorem

$$
\begin{aligned}
\oint_{\gamma} H(z) d z & =\oint_{\gamma} \int_{\mathbb{R}} e^{x z} \mathbb{E}\left[\sum_{i=1}^{N}\left(1-\phi\left(e^{-x} A_{i}\right)\right)-\left(1-\phi\left(e^{-x}\right)\right)\right] d x d z \\
& =\int_{\mathbb{R}} \oint_{\gamma} e^{x z} d z \mathbb{E}\left[\sum_{i=1}^{N}\left(1-\phi\left(e^{-x} A_{i}\right)\right)-\left(1-\phi\left(e^{-x}\right)\right)\right] d x=0 .
\end{aligned}
$$

Therefore, by Morera Theorem $H$ is holomorphic in $0<\Re z<\alpha+\varepsilon_{0}$ and

$$
\begin{aligned}
H^{\prime}(\alpha) & =\lim _{h \rightarrow 0} \int_{\mathbb{R}} \frac{e^{x h}-1}{h} e^{x \alpha} \mathbb{E}\left[\sum_{i=1}^{N}\left(1-\phi\left(e^{-x} A_{i}\right)\right)-\left(1-\phi\left(e^{-x}\right)\right)\right] \\
& =\int_{\mathbb{R}} x e^{x \alpha} \mathbb{E}\left[\sum_{i=1}^{N}\left(1-\phi\left(e^{-x} A_{i}\right)\right)-\left(1-\phi\left(e^{-x}\right)\right)\right]=\int x G(x) d x,
\end{aligned}
$$

where the second equality follows from $\left(e^{x h}-1\right) / h \leq C e^{\varepsilon|x|}$ and the Lebesgue Theorem. 
Now take $z$ such that $\Re z<\alpha$ and observe that for any $a>0$

$$
\begin{aligned}
\int_{\mathbb{R}} e^{z x}\left(1-\phi\left(e^{-x} a\right)\right) d x=\int_{\mathbb{R}} e^{z x} \mathbb{E}\left[\int_{0}^{e^{-x} a R} e^{-u} d u\right] d x \\
=\mathbb{E}\left[\int_{0}^{\infty} e^{-u} \int_{e^{x}<a R / u} e^{z x} d x d u\right]=\mathbb{E}\left[\frac{a^{z} R^{z}}{z} \int_{0}^{\infty} e^{-u} u^{-z} d u\right] \\
=\frac{a^{z} \mathbb{E}\left[R^{z}\right] \Gamma(1-z)}{z}<\infty .
\end{aligned}
$$

Therefore for $\Re z<\alpha$ we can express $H$ in another form:

$$
\begin{aligned}
H(z) & =\int_{\mathbb{R}} e^{z x} \mathbb{E}\left[\sum_{i=1}^{N}\left(1-\phi\left(e^{-x} A_{i}\right)\right)-\left(1-\phi\left(e^{-x}\right)\right)\right] d x \\
& =\frac{\mathbb{E}\left[R^{z}\right]\left(\mathbb{E}\left[\sum_{i=1}^{N} A_{i}^{z}\right]-1\right) \Gamma(1-z)}{z}=\frac{\mathbb{E}\left[R^{z}\right](m(z)-1) \Gamma(1-z)}{z} .
\end{aligned}
$$

From this we obtain that for $0<\Re z<\alpha$

$$
\mathbb{E}\left[R^{z}\right]=\frac{z H(z)}{(m(z)-1) \Gamma(1-z)}
$$

However, if we assume that both $H(\alpha)=\int G(x) d x$ and $H^{\prime}(\alpha)=\int x G(x) d x$ are equal to 0 , then $\alpha$ is a root of multiplicity at least two of $H$. By our assumptions $m(\alpha)-1=0, m^{\prime}(\alpha)=0$. The strong convexity of $m$ yields $m^{\prime \prime}(\alpha)>0$. Since $\Gamma(1-\alpha) \neq 0$ the right side of (3.32) defines a holomorphic function on $0<\Re z<\alpha+\varepsilon_{0}$ that extends $\mathbb{E}\left[R^{z}\right]$. From the Landau Theorem we know that if $\mathbb{E}\left[R^{z}\right]$ has a holomorphic extension on $\Re z<\alpha+\varepsilon_{0}$ then it is expressed by the same formula $\mathbb{E}\left[R^{z}\right]$. In particular, one can find positive $\delta^{\prime}$ such that $\mathbb{E}\left[R^{\alpha+\delta^{\prime}}\right]<\infty$, contrary to the Corollary 3.18.

\section{REFERENCES}

[1] E. Aïdékon and Z. Shi. Weak convergence for the minimal position in a branching random walk: a simple proof. Period. Math. Hungar, 61(1):43-54, 2010.

[2] D.J. Aldous and A. Bandyopadhyay. A survey of max-type recursive distributional equations. Ann. Appl. Probab., 15(2):1047-1110, 2005.

[3] G. Alsmeyer, J.D. Biggins, and M. Meiners. The functional equation of the smoothing transform. To appear in Ann. Probab.

[4] G. Alsmeyer and M. Meiners. Fixed points of the smoothing transform: Two-sided solutions. Probab. Theory Related Fields, pages 1-35.

[5] G. Alsmeyer and M. Meiners. Fixed points of inhomogeneous smoothing transforms. J. Differ. Equations Appl., 18(8):1287-1304, 2012.

[6] M. Babillot, P. Bougerol, and L. Elie. The random difference equation $x_{n}=a_{n} x_{n-1}+b_{n}$ in the critical case. Ann. Probab., 25(1):478-493, 1997.

[7] F. Bassetti, L. Ladelli, and G. Toscani. Kinetic models with randomly perturbed binary collisions. J. Stat. Phys., 142(4):686-709, 2011.

[8] S. Brofferio, D. Buraczewski, and E. Damek. On the invariant measure of the random difference equation $x_{n}=$ $a_{n} x_{n-1}+b_{n}$ in the critical case. Ann. Inst. H. Poincaré Probab. Statist., 48(2):377-395, 2012.

[9] D. Buraczewski. On invariant measures of stochastic recursions in a critical case. Ann. Appl. Probab., 17(4):1245$1272,2007$.

[10] D. Buraczewski. On tails of fixed points of the smoothing transform in the boundary case. Stochastic Process. Appl., 119(11):3955-3961, 2009.

[11] D. Buraczewski, E. Damek, Y. Guivarch, A. Hulanicki, and R. Urban. Tail-homogeneity of stationary measures for some multidimensional stochastic recursions. Probab. Theory Related Fields, 145(3):385-420, 2009.

[12] D. Buraczewski, E. Damek, and J. Zienkiewicz. Precise tail asymptotics of fixed points of the smoothing transform with general weights. Preprint. 
[13] R. Durrett and T.M. Liggett. Fixed points of the smoothing transformation. Probab. Theory Related Fields, 64(3):275-301, 1983.

[14] W. Feller. An introduction to probability theory and its application, volume II. Wiley, 2nd edition, 1971.

[15] C.M. Goldie. Implicit renewal theory and tails of solutions of random equations. Ann. Appl. Probab., 1(1):126$166,1991$.

[16] Yueyun Hu and Zhan Shi. Minimal position and critical martingale convergence in branching random walks, and directed polymers on disordered trees. Ann. Probab., 37(2):742-789, 2009.

[17] P.R. Jelenković and M. Olvera-Cravioto. Information ranking and power laws on trees. Adv. in Appl. Probab., 42(4):1057-1093, 2010.

[18] P.R. Jelenković and M. Olvera-Cravioto. Implicit renewal theory and power tails on trees. Adv. in Appl. Probab., 44(2):528-561, 2012

[19] H. Kesten. Random difference equations and renewal theory for products of random matrices. Acta Math., 131(1):207-248, 1973.

[20] Q. Liu. Fixed points of a generalized smoothing transformation and applications to the branching random walk. Adv. in Appl. Probab., 30(1):85-112, 1998.

[21] Ralph Neininger and Ludger Rüschendorf. A general limit theorem for recursive algorithms and combinatorial structures. Ann. Appl. Probab., 14(1):378-418, 2004.

[22] Mathew D. Penrose and Andrew R. Wade. On the total length of the random minimal directed spanning tree. Adv. in Appl. Probab., 38(2):336-372, 2006.

[23] D. Revuz. Markov Chain. North-Holland Math. Library, 2nd edition, 1984.

[24] U. Rösler and L. Rüschendorf. The contraction method for recursive algorithms. Algorithmica, 29(1-2):3-33, 2001. Average-case analysis of algorithms (Princeton, NJ, 1998).

[25] F. Spitzer. Principles of Random Walk. Springer, 2nd edition, 1976.

[26] Y. Volkovich and N. Litvak. Asymptotic analysis for personalized web search. Adv. in Appl. Probab., 42(2):577$604,2010$.

[27] D. V. Widder. The Laplace Transform. Princeton University Press, Princeton, 1946.

D. Buraczewski, K. Kolesko, Instytut Matematyczny, Uniwersytet Wroclawski, 50-384 Wroclaw, pl. Grunwaldzki 2/4, Poland

E-mail address: dbura@math.uni.wroc.pl, Konrad.Kolesko@math.uni.wroc.pl 